\title{
Von Neumann e a previsão numérica de tempo e clima
}

\author{
Antonio Divino Moura
}

\begin{abstract}
Aercepção de John von Neumann de que a Meteorologia seria uma das áreas que mais se beneficiaria com o advento da 1 técnica de computação digital rápida e programável faz com que
hoje a sociedade se beneficie de previsões de tempo acuradas para 24,48 , até cerca de 120 horas, em nível mundial. Tal fato pode ser contrastado com as previsões precárias de 40 anos atrás, quando teve início a moderna era de previsão numérica de tempo através do Joint Numerical Weather Prediction Unit, mais tarde National Weather Service americano. Atualmente as previsóes para 24 horas sobre os Estados Unidos atingem $97 \%$ de índice de acerto com um modelo de área limitada de $70 \mathrm{~km}$ de resolução, 50 níveis na vertical e passos de integração de $2 \mathrm{~min}$, contrastado com o modelo barotrópico (um só nível) e $750 \mathrm{~km}$ de resolução.
\end{abstract}

Os fatos que ocorreram entre 1942 e 1955, com a influência direta de von Neumann, foram marcantes para transformar a Meteorologia, cuja atividade de previsão de tempo mais se aproximava de arte do que de ciência. Von Neumann, quando teve uma conversa com Carl-Gustav Rossby, então o líder mundial da Meteorologia Dinâmica, na Universidade de Chicago, ficou chocado ao saber que a previsão de tempo ainda dependia da destreza de cada previsor e estava longe de realmente ser um ramo da Física aplicada, como deveria ser. Por exemplo, os meteorologistas alemães e americanos divergiram muito sobre a previsão para o Dia D da invasão da Normandia por forças aliadas. Os previsores alemães disseram a Rommel que o tempo, no dia 5 de junho de 1944, estaria tão ruim que ele não deveria se preocupar com uma invasão.

A formação do grupo de meteorologistas liderados por Jule Gregory Charney, recém-doutor orientado de Holmboe na. UCLA, foi fundamental para que se produzisse a primeira previsão numérica de tempo bem sucedida no computador ENIAC, localizado na U.S. Army Aberdeen Proving Ground, em Maryland. Esse trabalho, em co-autoria com Ragnar Fjortoft, professor visitante norueguês, e von Neumann revolucionou, em 1950, a história da previsão de tempo e deve ser considerado com 
um marco, um divisor de águas, da Meteorologia moderna. Realmente, dava-se início à fase mais científica desta área da Física aplicada.

Não satisfeito, von Neumann foi além e percebeu ser-também a oportunidade para se atacar o problema da Climatologia via modelagem e simulação por modelos globais da atmosfera e dos oceanos. Von Neumann reconheceu claramente que o trabalho de Norman Alton Phillips, também do grupo de Charney, ao simular características da circulação atmosférica com integrações longas (cerca de 30 dias) no ENIAC do Instituto para Estudo Avançado, na Universidade de Princeton, dava início também à possibilidade de previsão climática.

Como conseqüência, o internacionalmente conhecido GFDL (Geophysical Fluid Dynamics Laboratory) mantido pela NOAA (National Oceanic and Atmospheric Administration), no campus da Universidade de Princeton, foi obra inicial de von Neumann, que assegurou fundos para o projeto em 1955. O GFDL é uma das instituições líderes nas simulações das possíveis alterações climáticas devido ao aumento na concentração de gás carbónico, fruto da ação antropogênica desregrada. Com certeza, um gênio como Jöhn von Neumann lançaria forte luz científica na solução adequada desta questão complexa de global change com que nos confrontamos. Mas, paciência, ele já fez muito!

\section{A Meteorologia operacional com computadores - 1955}

Em julho de 1955, nos Estados Unidos, o Weather Bureau, o Air Weather Service e o Naval Weather Service decidiram formar a Joint Operational Numerical Weather Prediction Unit, que começou a produzir rotineiramente mapas de previsão computadorizados, em setembro do mesmo ano, com um computador IBM 701, adquirido em março. Tal evento, segundo Meteorology and America 1920-1995, marca a história da utilização operacional do computador na previsão do tempo e no desenvolvimento de modelos e técnicas numéricas para a sua solução. $O$ primeiro modelo utilizado tomava uma representação simplística da atmosfera, em um só nível $(5 \mathrm{~km})$ e explicava somente $65 \%$ da variabilidade dos padrões característicos das condições atmosféricas em escala planetária. Por trás de toda essa revolução tecnológica/meteorológica estava von Neumann e vários colaboradores, talentosos meteorologistas, em Princeton, no Instituto para Estudo Avançado.

Para melhor apreciar tal feito, e entender o gênio de von Neumann e o enorme talento de vários de seus colaboradores em Meteorologia, entre 
eles Jule Charney, Fjortoft, Norman Phillips, Joseph Smagorinsky, para mencionar alguns, vamos voltar alguns anos atrás. O início de previsão numérica de tempo toma por base o trabalho pioneiro de von Neumann (Charney, et al., 1950), como ver-se-á adiante.

\section{O sucesso do experimento de Charney, Fjortoft e von Neumann - 1950}

Em 1946, ao final da Segunda Guerra Mundial, realizou-se uma Conferência sobre Matemática na Universidade de Princeton (Problems of Mathematics, 1947). Charney (1947) relata que, nesta conferência, von Neumann afirmou que o sucesso da Matemática com as equações diferenciais lineares da Eletrodinâmica e da Mecânica Quântica havia dissimulado suas falhas com as equações diferenciais não-lineares da Hidrodinâmica, Elasticidade e da Relatividade Geral. Von Neumann expressou sua esperança de que as soluções obtidas através de computador, para uma ampla gama de problemas em Mecânica do contínuo, não-linear, forneceria, sem dúvida, base para generalizações. Para von Neumann, relata Charney, Meteorologia era, por excelência, o ramo aplicado da Matemática e da Física que se candidataria a ganhar muito com o advento da computação de alta velocidade. E esta visão de há 50 anos certamente confirmou-se correta!

Naquele mesmo ano, von Neumann convocou uma reunião de líderes da área de Meteorologia para anunciar o projeto de um computador eletrônico, de aplicação geral, que estava construindo no Instituto para Estudo Avançado, Universidade de Princeton, buscando sua participação, aconselhamento e assistência em projetar os programas meteorológicos para o seu uso.

Carl-Gustav Rossby (1939), líder mundial em Meteorologia Dinâmica, participou da reunião, conforme descreve Charney, interpretava o consenso dos presentes e, apesar do interesse demonstrado, mostrava sinais de pouco entusiasmo. Para Rossby, o problema matemático ainda não estava totalmente definido: havia mais variáveis do que equações, já que não se havia sido capaz de exprimir os tensores tensão de Reynolds em termos das variáveis descritivas do escoamento médio. Rossby citou o caso da tentativa frustrada de Richardson et al. (1922), que calcularam, em 1920, por meio numérico e com os dados existentes, a tendência da pressão atmosférica $(\delta p / \delta t)$ de uma coluna de ar sobre a Europa. O resultado, após seis meses de cálculos, foi desastroso: Richardson indicou o valor de $+145 \mathrm{mb} / 6$ horas para $\mathrm{o}$ instante $t=0$, quando o encontrado 
foi de somente $+0,2 \mathrm{mb} / 6$ horas no intervalo anterior a $t=0$ e de $-1,0$ $\mathrm{mb} / 6$ horas no intervalo posterior a $t=0(t=0$ é identificado com o dia internacional de balóes sobre a Europa, em 1910, durante o qual obtiveram dados de ar superior).

Rossby afirmou que os cálculos não seriam bem sucedidos antes que melhores observações da atmosfera, análise e experimentação conduzissem ao melhor entendimento dos processos fundamentais operantes na atmosfera, em particular aqueles relacionados com a turbulência. A cautela de Rossby teve o efeito positivo de convencer von Neumann para a necessidade de análise física em adição à matemática. No entanto, Rossby falhou em não apreciar o enorme estímulo psicológico que a possibilidade de utilização da capacidade de efetuar cálculos numéricos em alta velocidade traria para a Meteorologia, tanto operacional como em pesquisas.

No ano de 1946; von Neumann havia negociado um contrato meteorológico com a Marinha Americana (neste contrato von Neumann expressou seu entusiasmo quanto a computação em alta velocidade abrir novas possibilidades para se estudar a circulação estratosférica e a turbulência atmosférica, e... faria com que a previsão de tempo uma semana ou mais adiante tornar-se-ia prática). Para os entusiastas, este foi o início da moderna era da Meteorologia, enquanto para os incrédulos, somente um projeto visando a obter apoio para cinco estudantes de pós-graduação passarem dois anos em Princeton trabalhando no porquê da falha de Richardson (MacRae, 1992).

O grupo de meteorologistas no Instituto para Estudo Avançado (IAS) não se conformou, de fato, até a chegada de Charney em 1948. O trabalho de doutorado de Charney e sua análise de escala dos movimentos atmosféricos mostram nova abordagem para a obtenção de um conjunto consistente de equações de previsão, a partir da derivação de uma hierarquia de complexidade crescente e consistente de modelos energeticamente consistentes.

A ideia básica de Charney seria a de utilização de modelos mais simples para explicar casos já observados e crescer em complexidade de modelo caso não se pudesse prever adequadamente o evento observado.

Charney já tinha um modelo simples barotrópico (neste, o escoamento atmosférico no nivel entre 3 a $6 \mathrm{~km}$ comporta-se como em duas dimensões - nos sentidos latitudinal e meridional) quase pronto para implementar no ENIAC (Electronic Numerical Integrator and Calculator) 
e que ficaria pronto entre 1948-49 em Princeton, no IAS. Como não foi terminado em tempo hábil, o ENIAC, já funcionando em Aberdeen, Maryland, com a adição de uma memória por von Neumann, foi utilizado por 33 dias, a partir de 6 de março de 1950 . Neste experimento, a previsão (para o dia 31 de janeiro de 1949) foi muito boa e tomou cerca de 36 horas de tempo do ENIAC para calcular outra, de 24 horas (cálculos que tomariam cerca de oito anos numa calculadora de mesa!). Charney sabia que o ENIAC (em construção) de Princeton seria mais rápido quando ficasse pronto, e tal fato não o perturbou. No entanto, outros exemplos de previsão não muito bem-sucedidos chamavam a atenção de Charney e de seu grupo: alguma baroclinia (significando que a atmosfera varia suas propriedades com a altura) deveria ser inserida nos modelos futuros (Charney, 1955).

O primeiro modelo de Charney, Fjortoft e von Neumann consistiu na solução matemática e na codificação numérica para a solução da equação da vorticidade barotrópica quase-geostrófica:

$$
\frac{\delta Z}{\delta t}=\left(\frac{1}{a \cos \phi}\right)^{2} \frac{\delta(Z, \psi)}{\delta(\phi, \lambda)}
$$

onde $\delta / \delta t$ significa derivada parcial com o tempo, e $Z=$ Laplaciano $\psi+$ $2 \Omega \sin \phi$, a vorticidade quase-gestrófica e $\psi(\phi, \lambda, t)$ a função de corrente sobre uma esfera em rotação $(\Omega)$, raio $a$, latitude $\phi$ e longitude $\lambda$.

A solução proposta no trabalho foi a de calcular as variáveis no tempo $t$, levando os resultados ao tempo $t+\delta t$, através dos seguintes passos, começando com $\psi(t-\delta t)$ e $\psi(t)$ :

(1) calcular $\delta Z / \delta t$ pela equação acima $\mathrm{e}$

(2) depois para $\psi$, resolvendo a equação de Poisson, então resolver

(3) $\psi(t+\delta t)=\psi(t-\delta t)+2 \delta t(\delta \psi / \delta t)$, este último calculado em $t=t$. (vide Charney \& Phillips (1953) para esta solução).

Este modelo - trata-se na realidade de um caso particular do Teorema de Ertel (1942) para a vorticidade potencial, que é conservada para escoamento invíscidos e adiabáticos, conforme também trabalho de Rossby (1939) - e a sua metodologia de solução conseguiram superar as dificuldades encontradas por Richardson em 1920, uma vez que, através do balanço quase-geostróficos da atmosfera em larga escala, consegue 
filtrar o denominado ruído atmosférico introduzido pelas ondas de gravidade (de alta velocidade) presentes no modelo completo mais sofisticado utilizado por ele. Hoje existem técnicas numéricas e de inicialização dos modelos de previsão que evitam tais desequilíbrios e tendências elevadas, não-realistas para a pressão atmosférica dos sistemas sinópticos observados e responsáveis pelo tempo do dia-a-dia, como os ciclones e as frentes frias a eles associadas.

\section{O experimento de Phillips para a simulação do clima - 1955}

Quando o computador ENIAC de von Neumann ficou pronto em Princeton, Norman Phillips juntou-se ao grupo de meteorologia, em setembro de 1951, a convite de Charney que o havia visitado na Universidade de Chicago (Taba, 1955) para ouvi-lo sobre sua tese de doutorado com George Platzman e a utilização de um modelo baroclínico de duas camadas. (Em 1953, Phillips esteve em Estocolmo, no Instituto Meteorológico Internacional, estabelecido por Rossby, contando com o apoio do Escritório de Pesquisa Naval americano, onde começaram a programar o computador BESK, também com um modelo barotrópico - os suecos conseguiram efetuar previsão numérica em 1954, em tempo hábil para utilizá-la). Phillips relata que havia urgência para que os resultados das previsões ficassem prontos até dezembro de 1953, quando von Neumann gozaria férias com sua esposa. O grupo sueco realizou as previsões e enviou-lhe um telegrama informando os resultados; Phillips acredita ter adicionado alegria às férias de von Neumann!

Phillips (1953) conseguiu realizar longa integração com um modelo quase-geostrófico baroclínico simples de duas camadas, com condição inicial de repouso (ventos nulos), incluindo efeitos realísticos de atrito e de aquecimento. $O$ domínio de integração foi um retângulo de $17 \times 16$ pontos, com resolução de $375 \mathrm{~km}$ na direção $x$ (longitude), para o número de onda 4 mais instável, e $625 \mathrm{~km}$ na direção $y$ (latitude), do equador ao pólo.

Os resultados mostram com realismo a evolução das perturbações átmosféricas, simulando o aparecimento dos ciclones e anticiclones, com escalas temporais e espaciais próximas das observadas. A energética do modelo e das perturbações obtidas é demonstraida em detalhe neste trabalho, o qual deu origem à possibilidade de simulação climática utilizando os mesmos princípios da Mecânica de Fluidos e da Termodinâmica, como mostrado por Bjerknes (1904). Trata-se de trabalho marcante na área 
da Climatologia Dinâmica, realizado em seu laboratório de simulação climática, testando hipóteses realistas sobre o comportamento do fluido atmosférico em escala planetária.

\section{O surgimento do GFDL - 1955 e 1963}

Smagorinsky (1963) também esteve envolvido com os trabalhos do grupo de meteorologia, mas como meteorologista do Weather Bureau. Conta, em detalhes (Smagorinsky (1983)), como os resultados obtidos por Phillips foram encorajadores, a ponto de von Neumann propor uma grande e nova iniciativa, separada e dedicada. Von Neumann elaborou uma proposta ao Weather Bureau, à Força Aérea e à Marinha justificando um projeto conjunto para o estudo da dinâmica da circulação atmosférica (ver anexo parte do rascunho, com anotações de punho de von Neumann, para este projeto). A proposta é datada de 1 de agosto de 1955 e foi aceita para ser iniciada um mês depois como uma atividade conjunta Weather Bureau-Air Force-Navy. Coube a Smagorinsky liderar a nova Seção de Pesquisa sobre a Circulação Geral. No final daquele ano o grupo estava constituído por outros quatro pesquisadores.

Na primavera (hemisfério norte) de 1955 von Neumann mudou-se para Washington, exercendo a função de Commissioner of Atomic Energy e manteve contato constante com o grupo de Smagorinsky até sua trágica doença, em 1956, que causou sua morte prematura em 1957, com 53 anos de idade.

Como resultado da ida de von Neumann para Washington, a indiferença latente ao projeto do computador por parte do Instituto para Estudo Avançado acentuou-se, fazendo com que Charney e Phillips deixassem a Instituição e buscassem nova afiliação com o MIT. Assim, a Força Aérea e a Marinha retiraram seu apoio ao projeto, sendo este somente apoiado pelo Weather Bureau. Em 1953 Smagorins;ky publicou o resultado de seu trabalho detalhado, com um modelo de equações primitivas, baroclínico de nove níveis na vertical.

Começou assim em Washington um dos centros de maior respeito científico internacional nos dias de hoje - o GFDL (Geophysical Fluid Dynamics Laboratory), mantido pela NOAA (National Oceanic and Atmospheric Administration). O fato ocorreu, em parte, também devido à integração ao grupo de cientistas de dois expoentes da pesquisa meteorológica japonesa, em outubro de 1959, Sikuro Manabe e Kikuro Myakoda. A designação formal GFDL surgiu em 1963; em 1968 mudou-se de 
Washington para Princeton, onde permanece. Smagorinsky permaneceu como diretor do Laboratório até 1984, quando aposentou-se.

Manabe é considerado hoje um dos mais respeitados pesquisadores na questão do efeito estufa adicional, com modelos complexos que compreendem, de modo iterativo e acoplado, os dois fluidos planetários: a atmosfera e os oceanos, com simulações que cobrem períodos de centenas a milhares de anos, com desempenho realista e que servem de base para o conhecimento e a tomada de decisão sobre a questão de global change.

Surgiu assim, pelas mãos e visão genial de von Neumann, a nova área de Climatologia, com os modelos numéricos sendo a ferramenta fundamental para simulações e testes de hipóteses. Trata-se, portanto, do laboratório moderno do climatologista preocupado com a variabilidade natural do clima, as mudanças prováveis pela ação antropogênica neste século, a excessiva produção de gás carbônico nas indústrias e no transporte rodoviário, utilizando combustíveis fosseis. São novos desafios e seria muito confortante se pudéssemos contar hoje com a visão e o gênio de von Neumann na busca de soluções não somente do ponto de vista científico, mas para o benefício da humanidade.

Referências bibliográficas

Meteorology and America 1920-1995. Weatherwise, Boston, MA, American Meteorological Society, June/July 1995.

BJERKNES, V. Das problem von der wettervorhersage, betrachtet von standpunkt der mechanic und der physik. Meteorol. Z., n. 54, p. 1-22, 1904.

CHARNEY, $\mathrm{J}$. The use of primitive equations of motion in numerical prediction. Tellus, v. VII, p. 22-26, 1955.

. The dynamics of long waves in a baroclinic westerly current. Journal of Meteorology, v. 4, n. 5, 1947, p. 135-162.

Impact of computers on meteorology. Computer Physics Communications 3, suppl., p. 117-126.

n. XVII, 2, 17 p. 
CHARNEY, J. G.; FJORTOFT, R. \& VON NEUMANN, J. Numerical integration of the vorticity equation. Tellus, n. 2, p. 237-254, 1950.

CHARNEY, J. G. \& PHILLIPS, N. A. Numerical integration of the quasigeostrophic equations for barotropic and simple baroclinic flows. Journal of Meteorology, v. 10, n. 2, p. 71-99, 1953.

ERTEL, H. Ein neuer hydrodynamischer wirbelsatz. Meteorol. Z., n. 59, p. $277-281,1942$.

MACRAE, N. John von Neumann. New York, Pantheon Books, 1992. 406 p.

PHILLIPS, N. The general circulation of the atmosphere: a numerical experiment. Quarterly Journal of the Royal Meteorological Society, v. 82, p. $123-164,1956$.

Problems of Mathematics. Series 2 of Princeton University Bicentennial Conferences, 1947.

RICHARDSON, L. F. Weather prediction by numerical process. London, University Press, 1922. 236p. Também como New York, Dover Publication, 1965.

ROSSBY, C.-G. \& et al. Relations between variations in the intensity of the zonal circulation of the atmosphere and the displacements of the semipermanent centres of actions. Journal of Marine Research, v. 2, p. 212-229, 1939.

SMAGORINSKY, J. General circulation experiments with the primitive equations. I. The basic experiment. Monthly Weather Review, v. 91, p. 99-164, 1963.

The beginnings of numerical weather prediction and general circulation modeling: early recollections. Advances in Geophysics, v. 25, p. 3-37, 1983.

TABA, H. Interview with Norman Phillips. Bulletin of the World Meteorological Organization 1995.

Leituras adicionais

PLATZMAN, G. W. Some remarks on high-speed automatic computers and their use in meteorology. Tellus, n. 4, p. 168-178, 1952. 
PLATZMAN, G. W. A retrospective view on Richardson's book on weather prediction. Bulletin of the American Meteorological Society, v. 48, 1967.

PHILLIPS, N. Models for weather prediction. annual review of fluid-mechanics. Annual Review of Fluid Mechanics, v. 2, p. 251-292, 1970.

LEMES, M. A. M. \& MOURA, A. D. Previsão numérica de tempo e sua perspectiva no Brasil. INPE-1670-RPE/108. Apresentado na 31a. Reunião Anual da SBPC, Fortaleza, julho 1979.

Antonio Divino Moura é pesquisador do Instituto Nacional de Pesquisas Espacias (INPE).

Palestra feita pelo autor no encontro A obra e o legado de John von Neumann, organizado pelo Instituto de Estudos Avançados da USP e pela Academia Brasileira de Ciências no Instituto de Matemática e Estatística da USP em 14 de novembro de 1995 . 